\title{
An evaluation of oral health-related quality of life in orthodontic patients treated with fixed and twin blocks removable appliances: part I
}

\begin{abstract}
Objective: The study evaluated the impact of orthodontic treatment on the quality of life in two patient groups with Class II Division 1 malocclusion.

Materials and methods: 98 patients were assigned to Fixed or Twin-block appliance treatment groups. Patients' treatment needs were categorized with IOTN. The OHRQoL was assessed by OHIP-14 during different periods of treatment.

Results: Both groups showed comparable improvements in OHRQoL as the treatment progressed (Anova test $\mathrm{p}=0.05$ ). Similar results were observed when OHRQoL was analyzed at IOTN subgroup level (Bonferroni Test $\mathrm{p}<0.05$ ).
\end{abstract}

Conclusion: OHRQoL improved significantly with both Fixed and Functional appliances.
Volume 5 Issue 4 - 2016

\author{
Emad EM Alzoubi, Kevin Mulligan, Nikolai \\ Attard \\ Department of Dental Surgery, Faculty of Dental Surgery, \\ University of Malta, Malta
}

\begin{abstract}
Correspondence: Emad EM Alzoubi, Department of Dental Surgery, Faculty of Dental Surgery, University of Malta, Medical School, Mater Dei Hospital, Block A, Level O, B'Kara Bypass, Msida MSD2090, Malta, Email emad edd@hotmail.com
\end{abstract}

Received: October 26, 2015 | Published: November 18, 2016

\section{Introduction}

World Health Organization (WHO) in 1948, defined health as "a complete state of physical, mental, social wellbeing and not just the absence of the disease". This underscores the importance of quality of life defined as patients" "perceptions of their position in life in the context of culture and value systems in which they live, and in relation to their goals, expectations, standards, and concerns". ${ }^{1}$ A person's quality of life (QoL) can be affected with poor oral health. ${ }^{2}$ Measurement of oral health related quality of life (OHRQoL) helps professionals clarify the role of oral health status on the overall quality of life. ${ }^{3}$ It also assists in clinical decisions taking into account patients' needs and serves as an effective communication mechanism with policy makers. ${ }^{4,5}$ Consequently modern healthcare systems should address patient's health complaints, taking into consideration the impact of patients' illness on quality of life. ${ }^{6}$ Modern dental procedures Endeavour to improve patients' quality of life. Orthodontic treatment based on purely clinical and functional perceptions may not fully address patients' concerns. Patients and their parents have been shown to share similar treatment expectations, although parents reported more realistic prospects. Ethnicity significantly influences expectations for orthodontic treatment, and this may relate to differences in the patients' and parents' assessment of the clinical outcome. ${ }^{7}$ Although improvement of QoL is considered to be the major goal of orthodontic treatment, it is established that, to obtain enhancement in QoL, patients must go through some treatment-related undesirable side effects when using orthodontics appliances. ${ }^{8}$ Malocclusion is the most common oral disorder which can cause negative impacts on social life and self-confidence and may be greatly associated with negative psychological connotations. ${ }^{9}$ Studies reported that initially OHRQoL worsened during treatment; however a considerable improvement was observed afterwards. ${ }^{10-12}$ The aims of this prospective study were to assess the impact of two orthodontic treatment modalities, Fixed- and Twin block removable- appliances on patients' quality of life.

\section{Material and methods}

The study was conducted at the postgraduate university clinics within Mater Dei State Hospital. Research and ethical approvals for this study were granted from Mater Dei Hospital and University of Malta Research Ethics Committee. Patient recruitment is described in (Figure 1). Participation was on a voluntary basis; potential patients and their guardians were explained the study details and encouraged to raise any concerns. Participants could withdraw at any point in the study without affecting their treatment. Interested patients/ guardians were provided with a patient information package in their preferred language that is Maltese or English. Written consent was obtained. The clinical steps involved for the fixed appliances and Twin blocks removable appliances were explained to the parents/ guardians. In cases involving extractions, further explanation and specific was obtained for the procedure. Inclusion criteria involved medically fit school children aged 10-16 years, who were nonsyndromic, fully compliant, required simple extractions and had no previous orthodontic treatment. Exclusion criteria included patients below 10 years, and cases requiring headgear devices and surgical removal of teeth. Patients' occlusions for both groups were Class 2 division 1 incisors relationship ${ }^{13}$. The fixed appliances group was treated with MIM brackets pre-adjusted straight wire appliance ( 0.022 X 0.028 'inch) whilst the second group received Twin block removable functional appliances. An IOTN-DHC (Dental Health Component) index main grade was used to categorize the patients' variety of treatment needs (e.g. differences in over jet, the presence of hypodontia, crossbites, open bites, impacted canines etc). We did not use IOTN-DHC to compare the severity for malocclusions. The principle clinician used the main grades of IOTN (DHC) to categorize each patient. Intra-examiner testing was performed. The investigator evaluated 10 casts/day allowing 20 minutes recess between each cast scoring. To verify the results a second experienced clinician evaluated the casts and an inter-examiner reliability test was carried out on randomly allocated 50 casts. The OHRQoL was measured with the 
OHIP-14 questionnaire. Patients completed the questionnaire in their preferred language ${ }^{14,15}$ five times: prior to treatment as a baseline (T0), after six weeks of fitting the appliances (T1), after 12 weeks (T2), 6 month (T3) and at the end of the treatment (T4). OHIP-14 scores follow a Likert scale: the lowest score is Never $=1$, Hardly ever $=2$, occasionally $=3$, fairly often $=4$, Very often (Highest $)=5$.

\section{Statistical analyses}

1. The following statistical tests were applied:

2. Sample size and power analyses were estimated using Analysis of Covariance (ANCOVA).

3. Intra examiner and inter examiner reproducibility were estimated using weighted concordance Kappa's index.

4. Chi-square independence test and T-test were used to study homogeneity of treatment groups.

5. Kolmogorov-Smirnov's test was applied to assess normality of OHIP-14 global and sub-scores, at the different time-points.

6. A General Linear Model (GLM) for repeated measurements Analysis of Variance (ANOVA and Bonferroni Tests) was estimated to test differences of the means of an OHIP-14 outcome for the different follow-up periods according to the appliance group and IOTN index.

Significance level used in analyses was set at a p value of $<0.05$.

\section{Results}

The sample consisted of 98 subjects classified in two balanced groups according their orthodontic appliance. The gender distribution included 20 males and 29 females (Fixed group) and 21 females and 28 males (Functional group). A Chi2 test concluded that statistical differences were not reached $(\mathrm{p}=0.103)$. There were no significant differences in the average ages according to gender (T-Test $\mathrm{p}>0.05$ ). The ages were $13 \pm 1.18$ for males and $12.4 \pm 1.24$ years old for females. The observation period was around 25 months. The intra-examiner reliability for the classification of IOTN grades was estimated as good with a weighted Kappa's index of 0.73 . The inter-examiner reliability for the classification of IOTN grades was estimated as good with a weighted Kappa's index of 0.69.The IOTN-DHC indicated that most patients were in the grade 4 or 5 categories. Figure 1 explores the distribution of the patients. Patients that received fixed appliances were almost exclusively grade 4 or 5 ; however, roughly a third of those in the functional group were classified in the IOTN grade 3. In this case, statistical significance was reached (Chi2 test $\mathrm{p}<0.001)$. Moreover extractions were exclusively in the fixed appliance group rates with $63.3 \%$ requiring removal of teeth. These differences between the two main groups reached statistical significance $(p<0.001$, Chi2 test). In the fixed appliance group, extractions were observed in the IOTN 4 $(43.5 \%)$ and $5(60.6 \%)$ sub-groups. The OHIP-14 scores showed no significant differences in quality of life when the two orthodontic groups were compared together (Anova $\mathrm{p}-0.108$ ). Moreover no interactions were identified over time $(\mathrm{p}=0.518)$, indicating that changes in OHIP scores throughout time were similar for each group. However it was noted that there were differences in the OHIP mean scores within the groups over time (Bonferroni Tests $\mathrm{p}<0.001$ ) generally the scores at the end of treatment were significantly lower than the initial and intermediate time points. The severity of treatment needs, as described by IOTN index was not associated with the patients' level of quality of life (ANOVA test $\mathrm{p}>0.05$ ). However, for all the three IOTN subgroups a significant difference was observed at $\mathrm{T} 4$ when compared to (T0), (Test Bonferroni's $\mathrm{p}<0.05$ ). OHIP-14 was significantly lower at T4 with respect to T0 within each appliance group and each IOTN grade. Thus both appliances showed improvement in quality of life (Figure 2). We also observed that scores increased until (T1), considered being a period of adaptation and then slopes became negative. These changes in Global OHIP score total throughout time were similar for each group and non significant (Anova $\mathrm{p}=0.518$ ), OHIP-14 Functional scores Figure 3 OHIP-14 Functional scores were significantly lower at $\mathrm{T} 4$ with respect to $\mathrm{T} 0$, within each appliance group and each IOTN grade. (Bonferroni Test: $\mathrm{p}<0.05$ ). With regards to the IOTN 4 group, there were significant differences between groups at $\mathrm{T} 1$, $\mathrm{T} 3$ and T4, with higher satisfaction recorded for the patients in the fixed group. (Bonferroni Test $\mathrm{p}<0.05$ ) OHIP-14 Psychosocial scores (Figure 4) A significant improvement in OHIP 14 Psychosocial scores was observed at T4 for IOTN subgroups 4 and 5 (Bonferroni Test's $\mathrm{p}<0.05$ ). In the case of IOTN 3, a significant difference at T4 was reached for Twin block group only (Bonferroni's test $\mathrm{p}<0.001$ ). At T4, OHIP Psychosocial score for the fixed group were significantly lower than those for the Functional one independently of IOTN severity. (Bonferroni Test $\mathrm{p}<0.05$ ).

\section{IOTN Classification by Group}

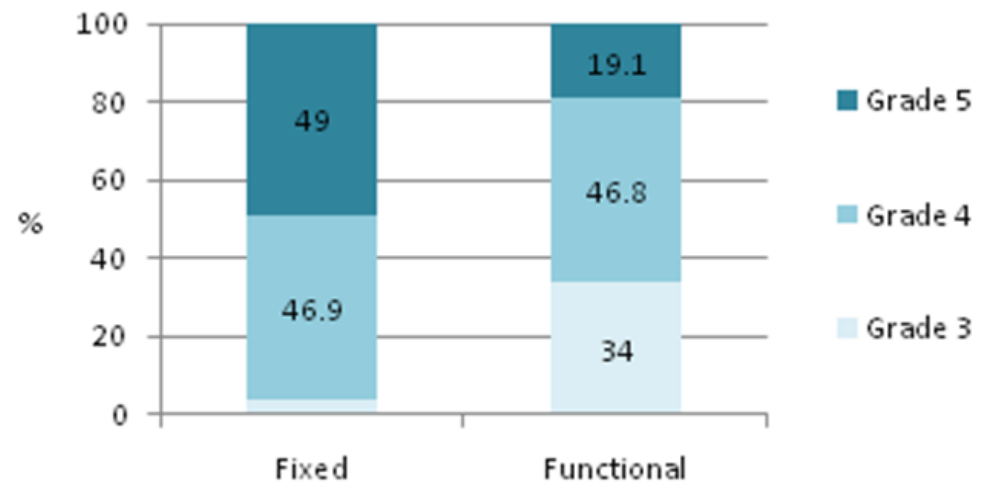

Figure I Percentage distribution of patients as per IOTN and type of appliance. oral health-related quality of life measurement. 
OHIP-14 Scores for IOTN Grades

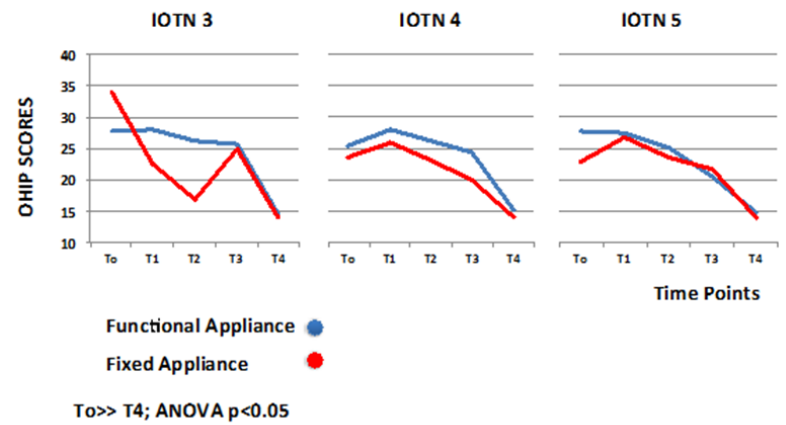

Figure 2 Changes in global OHIP-14 scores for the IOTN grades with time.

OHIP-14 Scores for IOTN Grades

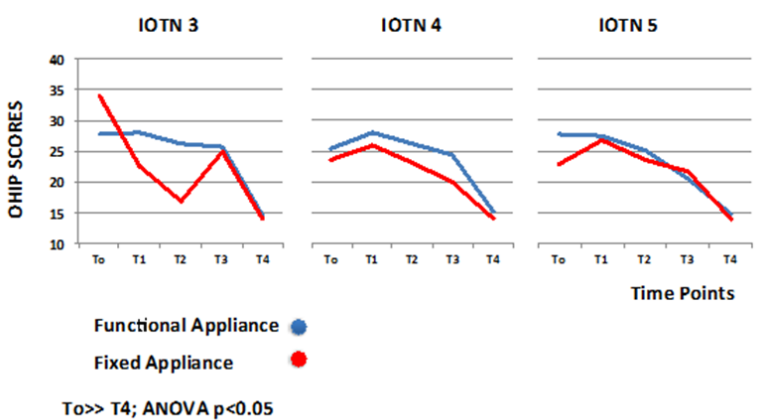

Figure 3 Changes in OHIP-I 4 functional scores for the IOTN grades with time.

\section{OHIP-14 Functional Scores for IOTN Grades}

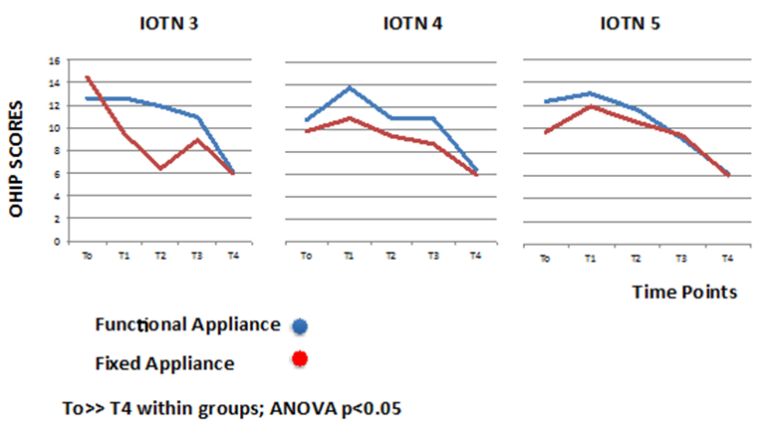

Figure 4 Changes in OHIP-I4 psychosocial scores for the IOTN grades with time.

\section{Discussion}

This prospective study showed that the quality of life improved significantly with Fixed and Twin block appliances by the end of treatment. They corroborate studies that observed that patient satisfaction and oral health-related quality of life improved significantly at the end of fixed orthodontic treatment ${ }^{16,17}$. To date no studies compared the changes in quality of life following treatment with Fixed and Twin block functional appliances. This study underscores the fact that quality of life improved significantly irrespective of the appliance and that the patients adapted and embraced treatment meaning that both appliances were equally effective. Chen et al. reported that fixed appliances deteriorate quality of life in the first month but then function and pain levels improved as treatment progressed..$^{18}$ It was reported that non-surgical tooth extraction prior to fixed appliance treatment could worsen the quality of life especially the functional aspects. ${ }^{19}$ We also observed similar trends. Extractions were a contributing factor, beside the fixed appliance itself, in worsening the quality of life at the beginning of the treatment as evidenced by the OHIP scores. Indeed the functional scores of OHIP 14 were high at T1 indicating worsened quality of life. Following healing and adaptation the quality of life improved remarkably. The fixed appliance group 
experienced more pain than the Twin Block group when eating, since the latter were asked to remove the appliance during meals. Authors reported that patients with removable appliances group experienced more issues with speech during treatment and had negative effects on schoolwork and leisure activities. ${ }^{20}$ This is due to the fact that removable appliances reduce and change the intraoral space, thus precluding the tongue from articulating certain speech sounds. In addition speech problems in the removable appliance group can also be a contributing factor to the negative effect on schoolwork and leisure activities reported. ${ }^{20}$ It is therefore not surprising that in our study the Twin block group demonstrated poorer quality of life at the beginning of the treatment since the appliance is bulky and hinders patients' speech. However we observed that once the patients adapted the quality of life improved. It is evident that both groups showed improvement in quality of life from baseline till the end of treatment. However it is important to remember the patient's own perceived expectation to the treatment outcome in relation to the presenting problem. Patients who used fixed appliances had all their teeth aligned to a high degree. On the other hand Twin block appliances group was treated to correct the over jet, with dento-alveolar and minor skeletal changes ( $\mathrm{O}^{\prime}$ brien $\mathrm{K}$ et al., 2003). Keeping in mind that patients seeking treatment expect different outcomes, it is no surprise that patients in the Twin block appliance group reported similar scores to the Fixed group. For example patients in the Twin block group, who were embarrassed with their teeth due to their malocclusion, would have perceived very favorably, a reduction of over jet of 11 $\mathrm{mm}$ to $3 \mathrm{~mm}$ by the end of treatment. The initial orthodontic treatment need (IOTN) scores were high at the beginning of the treatment but dropped significantly toward the end for fixed appliance group. The same effect was evident for the functional appliances group, though not as significant since the Twin blocks mainly addressed the over jet malocclusion at this stage of treatment. Most probably a further reduction in IOTN scores for the functional group would be observed if the same patients proceeded to fixed treatment. The sub-scores for the functional (IOTN 4) and psychosocial domains (IOTN 3, 4 5 ) were lower at the end of treatment for the fixed group. Although these results were statistically significant, it is pertinent to note that these results were clinically irrelevant. This study underscored the fact that IOTN was not sensitive in measuring orthodontic treatment needs from a patient's perspective or failed to address the patients chief complain. Rather it defined them from a clinician's point of view only. In this respect our study corroborated other authors' findings and highlighted the need for an index that looks holistically at the problem that is both from patient and clinician's perspectives. ${ }^{21}$ It is important to highlight that the costs incurred for these two treatment modalities can be different and therefore an economic analysis is warranted to investigate the cost effectiveness of treatment. This will be the subject of a second study.

\section{Conclusion}

All patients reported an improvement in their quality of life. At the end of treatment no statistically significant differences were found between the two groups.

\section{Acknowledgments}

None.

\section{Conflicts of interest}

The authors declare that there are no conflicts of interest.

\section{Funding details}

None.

\section{References}

1. World Health Organization. Preamble to the constitution of the World Health Organization as adopted by the International Health Conference. New York, 19-22 June, 1946; signed on 22 July 1946 by the representatives of 61 States (Official Records of the World Health Organization, no. 2, p. $100)$ and entered into force on 7 April, 1948.

2. Gift HC, Atchison KA. Oral health, health, and health-related quality of life. Medical care.1995;1(11 Suppl):NS57-NS77.

3. Cunningham SJ, Hunt NP. Quality of life and its importance in orthodontics. J Orthod. 2011;28(2):152-158.

4. Jokovic A, Locker D, Guyatt G. How well do parents know their children? Implications for proxy reporting of child health-related quality of life. Qual Life Res. 2004;13(7):1297-1307.

5. Al Shamrany M. Oral health-related quality of life: a broader perspective. East Mediterr Health J. 2006;12(6):894-901.

6. Sischo L, Broder HL. Oral Health-related Quality of Life What, Why, How, and Future Implications. J Dent Res. 2011;90(11):1264-1270.

7. Sayers MS, Newton JT. Patients' expectations of orthodontic treatment: Part 2-findings from a questionnaire survey. J Orthod. 2007;34(1):25-35.

8. Proffit WR, Fields HW, Sarver DM. Contemporary orthodontics. 5th ed. Elsevier Health Sciences; 2014.

9. Masood Y, Masood M, Zainul NN, et al. Impact of malocclusion on ora health related quality of life in young people. Health and quality of life outcomes. 2013;11:25.

10. Costa AA, Ferreira MC, Serra Negra JM, et al. Impact of wearing fixed orthodontic appliances on oral health-related quality of life among Brazilian children. J orthod. 2011;38(4):275-281.

11. Navabi N, Farnudi H, Rafiei $\mathrm{H}$, et al. Orthodontic treatment and the oral health-related quality of life of patients. $J$ Dentistry (Tehran). 2012;9(3):247-254

12. Feu D, Miguel JA, Celeste RK, et al. Effect of orthodontic treatment on oral health-related quality of life. The Angle Orthodontist. 2013;83(5):892-898.

13. British Standard Incisor Classification. Glossary of Dental Terms BS 4492. London: British Standard Institute; 1983.

14. Santucci D, Camilleri L, Attard N. Development of a Maltese version of oral health-associated questionnaires: OHIP-14, GOHAI, and the Denture Satisfaction Questionnaire. International Journal of Prosthodontics. 2014;27(1):44-49.

15. Mansor N, Saub R, Othman SA. Changes in the oral health-related quality of life $24 \mathrm{~h}$ following insertion of fixed orthodontic appliances. $J$ Orthod Sci. 2012;1(4):98-102.

16. Souki BQ, Figueiredo DS, de Abreu Lima IL, et al. Two-phase orthodontic treatment of a complex malocclusion: Giving up efficiency in favor of effectiveness, quality of life, and functional rehabilitation? American Journal of Orthodontics and Dentofacial Orthopedics. 2013;143(4):547-558

17. De Oliveira CM, Sheiham A. Orthodontic treatment and its impact on oral health-related quality of life in Brazilian adolescents. Journal of Orthodontics. 2004;31(1):20-27.

18. Chen M, Wang DW, Wu LP. Fixed orthodontic appliance therapy and its impact on oral health-related quality of life in Chinese patients. The Angle orthodontist. 2010;80(1):49-53. 
19. Adeyemo WL, Taiwo OA, Oderinu OH, et al. Oral health-related quality of life following non-surgical (routine) tooth extraction: a pilot study. Contemp clin Dent. 2012;3(4):427-432.

20. Wiedel AP, Bondemark L. A randomized controlled trial of self-perceived pain, discomfort, and impairment of jaw function in children undergoing orthodontic treatment with fixed or removable appliances. Angle Orthod. $2015 ; 86(2): 324-330$
21. Johal A, Cheung MY, Marcenes W. The impact of two different malocclusion traits on quality of life. British dental journal. 2007;202(2):E6. 